\title{
Sub-tenon Anaesthesia versus Intracameral Anaesthesia in Patients Undergoing Cataract Extraction: A Comparative Study of the Level of Pain, Visual Perception and Anxiety
}

Mushawiahti Mustapha, ${ }^{1}$ Muhammad Hazzril Hamzah, ${ }^{2}$ Soon Ken Chow, ${ }^{2}$ Nur Syuhada Zulkifli, ${ }^{2}$ Zalikha Abdul Latiff, ${ }^{2}$ Diana Melissa Dualim, ${ }^{2}$ Su Gaik Cheah, ${ }^{2}$ Mae Lynn Catherine Bastion. ${ }^{2}$

\begin{abstract}
Background: Phacoemulsification is a modern method of cataract extraction. Sub-tenon anaesthesia used to be the preferred anaesthetic technique for this procedure before intracameral anaesthesia gained its popularity in recent years. Nevertheless, many surgeons still believe that sub-tenon anaesthesia is better than topical anaesthesia. This study aimed to evaluate and compare the experiences of patients who were treated for cataract by phacoemulsification surgery using either sub-tenon or intracameral anaesthesia. Methods: Cross-sectional study conducted at Universiti Kebangsaan Malaysia Medical Centre, Kuala Lumpur, Malaysia. Participants were asked to complete a questionnaire within 1-2 hours following surgery. The questionnaires were designed to gather information on the patient's level of anxiety, visual perception and amount of pain experienced during cataract surgery. Results: A total of 62 patients were included in the study. Thirty-one patients received anaesthesia by sub-tenon injection, whereas another 31 patients received anaesthesia by intracameral injection. There were no significant differences in the level of pain experienced by the two groups of patients during instillation of anaesthesia and during surgery ( $p=0.205$ and $p=0.592$, respectively). There were also no significant differences in terms of visual perception and anxiety levels during surgery between the two groups ( $p=0.178$ and $p=0.731$, respectively). Conclusion: Intracameral anaesthesia obviates the need for an injection during cataract surgery and is as comfortable for patients as sub-tenon anaesthesia in terms of visual perception, level of pain, and anxiety.
\end{abstract}

Keywords: Phacoemulsification, Cataract Extraction, Anesthesia Local, Anxiety (Source: MeSH-NLM).

\begin{abstract}
Introduction
Phacoemulsification is a modern and popular method of cataract extraction. Sub-tenon anaesthesia, also known as parabulbar anaesthesia, pinpoint block, or episcleral block, is a popular alternative to peribulbar or retrobulbar anaesthesia. ${ }^{1,2}$ Sub-tenon space is a potential space between the capsule and the outermost layer of the eyeball, namely, the sclera. Local anaesthetics instilled into this space produces immediate analgesia and akinesia of the eyeball by diffusing posteriorly into the retro-orbital space to block the traversing sensory and motor nerves.
\end{abstract}

Intracameral anaesthesia is another method used in cataract surgery. It involves the injection of anaesthetics directly into the anterior chamber of the eye at the beginning of the surgery. It has gained popularity recently, with lidocaine being the commonest drug used for this purpose. ${ }^{3}$ The advantages of intracameral anaesthesia include the ease of application via a preexisting corneal wound. Many studies have been conducted to compare the level of pain during phacoemulsification with various techniques of local anaesthesia., ${ }^{2,47}$ Although intracameral anaesthesia is known to provide pain-free surgery, many surgeons still believe that regional anaesthesia via the sub-tenon route is better than topical anaesthesia. ${ }^{2,5}$
The aim of this study was to compare the level of pain experienced during phacoemulsification in patients receiving either intracameral or sub-tenon anaesthesia. Other variables, including the level of anxiety and visual perception, were also examined. Comparison between sub-tenon and intracameral anaesthesia in terms of anxiety and visual perception has not been reported in previous studies.

\section{Methods}

A cross-sectional study was conducted at the Universiti Kebangsaan Malaysia Medical Centre (UKMMC), Malaysia, from March to June 2010, and the protocol was approved by the ethics committee of UKMMC. Written informed consent was obtained from every patient participating in this study. The study subjects were men and women aged between 45-80 years old who were undergoing phacoemulsification surgery for cataracts. Patients included in the study were those willing to undergo routine cataract surgery performed by a trained ophthalmologist using either sub-tenon or intracameral anaesthesia. Exclusion criteria were as follows: subject age $<18$ years; known diagnosis of anxiety or psychiatric disorders; patients requesting or requiring sedation; and complicated or prolonged ( $>1$ hour) cataract surgery.

\footnotetext{
${ }^{1}$ Department of Ophthalmology, Faculty of Medicine, Universiti Kebangsaan Malaysia Medical Centre, Kuala Lumpur, Malaysia.
}

${ }^{2}$ Faculty of Medicine, Universiti Kebangsaan Malaysia Medical Centre, Kuala Lumpur, Malaysia. 
Patients were selected using universal sampling. All patients received standard preoperative medications, which included a combination of guttae tropicamide $0.8 \%$ (Alcon laboratories, USA) and guttae phenylephrine $5 \%$ (Alcon laboratories, USA) for pupil dilatation. Topical bupivacaine was instilled preoperatively to reduce the stinging sensation during cleaning with $5 \%$ povidone iodine. None of the patients received any preoperative or intraoperative sedation. Three experienced surgeons who routinely operated with sub-tenon anaesthesia and three other surgeons who routinely operated with intracameral anaesthesia performed the surgery for this study. The anaesthetic agents used were intravenous $2 \%$ lidocaine hydrochloride (lignocaine injection $2 \%$, Pfizer Ophthalmics, New York, USA) for the intracameral group and a combination of lidocaine and bupivacaine for the sub-tenon group. Phacoemulsification surgery was performed after routine preparation and draping and using routine methods. Complicated surgeries were excluded from the study.

\section{Results}

A total of 62 patients were included in the study. Thirty-one patients (mean age $68.6 \pm 6.7$ years) received intracameral local anaesthesia and 31 patients (mean age $67.3 \pm 7.2$ years) were given sub-tenon anaesthesia. Age difference was not statistically significant between the two groups $(P=0.497)$.

Most patients in this study reported no pain (Grade 0 ) or mild pain (Grade 1) during instillation of both types of anaesthesia $(P=0.205)$. Similarly, majority of the patients in both groups reported pain scores of grade 1 or o during surgery, and no significant difference was observed $(\mathrm{P}=0.592)$ (Table 1).

Patients in both groups reported seeing various types of images during the procedure, ranging from hand motions, lights, and various colours or combination of images. Six patients reported seeing no images at all. For the purpose of analysis, these various images were grouped based on the number of

Table 1. Comparison of Pain Level during Instillation of Anaesthesia and during Surgery.

\begin{tabular}{|c|c|c|c|c|}
\hline Pain Level & \multicolumn{2}{|c|}{ During instillation of anaesthesia } & \multicolumn{2}{|c|}{ During surgery } \\
\hline Grade 0 & $9(28.1)$ & $18(56.3)$ & $16(50.0)$ & $18(56.3)$ \\
\hline Grade 1 & $20(62.5)$ & $8(25.0)$ & $14(45.16)$ & $10(31.3)$ \\
\hline Grade 2 & $2(9.4)$ & $2(7.5)$ & $1(3.1)$ & $2(6.25)$ \\
\hline Grade 3 & Nil & $2(7.5)$ & Nil & $1(3.23)$ \\
\hline
\end{tabular}

Note: Mann-Whitney U test: During instillation of anaesthesia $\mathrm{p}=0.205$, During surgery $\mathrm{p}=0.592$.

All patients were requested to complete a questionnaire within 1-2 hours following their surgery. The questions mainly concerned the levels of pain, visual perception and anxiety levels experienced during instillation of anaesthesia and during surgery. In cases where the patient was illiterate or had difficulty in reading, the scales used in the questionnaire were explained and a verbal response was obtained.

Patients were asked to grade the levels of pain experienced during delivery of anaesthesia and during surgery on a visual analogue pain scale (VAS). Patients were asked to grade the pain they felt on a linear scale of 0 to 4 (grade $0=$ no pain, grade $1=$ mild pain, grade $2=$ moderate pain, grade $3=$ severe pain, grade $4=$ maximum imaginable pain). Patients were also asked about their visual perceptions during surgery.

Anxiety level was assessed using the State-Trait Anxiety Inventory (STAI). Calculations were conducted online. For the purpose of analysis, the scores were grouped into four categories: grade 1 (mild anxiety), grade 2 (moderate anxiety), grade 3 (severe anxiety) and grade 4 (very severe anxiety).

Statistical analysis was performed using SPSS 13.0 for Windows (SPSS Inc). Analyses of categorical data were performed using the Chi-square test. Comparison of the mean age between the two groups was conducted using the independent samples t-test, while comparisons for ordinal and other numerical variables were mainly conducted using the non-parametric Wilcoxon Mann-Whitney test. $\mathrm{p}$-values of less than 0.05 were considered statistically significant. images reported (Table 2, 3). Most patients in the sub-tenon group saw one to two images during surgery, with $28.12 \%$ of them seeing two images. For the intracameral group, majority of the patients $(40.62 \%$ ) saw two images, and $34.38 \%$ saw only one image. There were no significant differences between the two types of anaesthesia in the number of images reported $(P=0.178)$. Anxiety levels in the two groups were approximately equal, with more than $50 \%$ of patients in both groups reporting only mild (Grade 1) anxiety during surgery with no significant difference between the groups (Po.178) (Table 4).

Table 2. Qualitative Description of Visual Sensation during Surgery for Both Groups.

\begin{tabular}{lcc}
\hline Visual sensation & Sub-tenon [n (\%)] & Intracameral [n (\%)] \\
Nothing & $4(13 \%)$ & $2(6 \%)$ \\
\hline Light & $9(29 \%)$ & $6(19 \%)$ \\
Movement & $4(13 \%)$ & $3(11 \%)$ \\
Colours & $\mathrm{Nil}$ & $1(3 \%)$ \\
Light and movement & $14(45 \%)$ & $19(61 \%)$
\end{tabular}

Table 3. Number of Visual Sensation Reported by Patients.

\begin{tabular}{lcc}
\hline Number of images & Sub-tenon [n (\%)] & Intracameral [n (\%)] \\
Nothing & $4(12.5)$ & $2(6.3)$ \\
\hline 1 image & $14(43.8)$ & $11(34.4)$ \\
2 images & $9(28.1)$ & $13(40.6)$ \\
3 images & $5(15.6)$ & $1(3.1)$ \\
4 images & $\mathrm{Nil}$ & $5(15.6)$
\end{tabular}

Note: Mann-Whitney $\mathrm{U}$ test $\mathrm{p}=0.178$ 
Table 4. Comparison of Anxiety Level between the Sub-tenon and the Intracameral Group.

\begin{tabular}{lcc}
\hline Anxiety level & Sub-tenon [n (\%)] & Intracameral [n (\%)] \\
Grade 1 & $23(72)$ & $22(69)$ \\
Grade 2 & $7(22)$ & $7(24.3)$ \\
Grade 3 & $1(3.1)$ & $2(6.2)$ \\
Grade 4 & $1(3.1)$ & Nil
\end{tabular}

Note: Mann-Whitney $\mathrm{U}$ test $\mathrm{p}=0.178$.

\section{Discussion}

Numerous studies have reported various complications of regional anaesthesia in comparison with topical or intracameral anaesthesia during cataract surgery. ${ }^{4,8}$ Until recently, peribulbar anaesthesia was deemed to be the superior technique for cataract surgery as it allows for a less painful and less difficult surgery. ${ }^{9}$ However, papers published more recently suggest that regional anaesthesia is unnecessary for routine phacoemulsification. ${ }^{10,11}$ Pain is not the only significant factor when describing patient's experience during phacoemulsification. Visual analogue pain scale (VAS), which was used in this study, is known to provide reproducible results. ${ }^{12}$ In our study, patients receiving intracameral anaesthesia reported levels of pain similar to those reported by patients who received sub-tenon injection. The combination of preoperative topical bupivacaine administration and intracameral injection of lidocaine was found to be more superior than using topical anaesthesia alone. ${ }^{13}$ Iris manipulation and stretching of the uveal tissue can irritate patients under topical anaesthesia, and additional anaestheia is required in such circumstances. ${ }^{13}$

Intracameral anaesthesia utilises short acting lidocaine $2 \%$, whereas the combined regime of lidocaine and bupivacaine used for sub-tenon injection may have an anaesthetic effect that lasts for up to an hour. For this reason, difficult cataract cases for which duration of extraction surgery could potentially be longer may not be suitable candidates for intracameral anesthesia. Nevertheless, experienced surgeons may still opt for intracameral injection to avoid the unnecessary complications of regional anaesthesia and in view of the ease of instillation and cost-effectiveness.

STAI is a set of questionnaires used to assess the anxiety level in adults. The simplicity of the STAI instrument makes it ideal for this purpose, even for evaluating individuals with lower educational backgrounds. ${ }^{14,15}$ Adapted in more than forty languages, the STAI is the leading measure of personal anxiety worldwide. STAI contains 40 questions, each with four possible responses: not at all, somewhat, moderately so and very much so. Au Eong et al., reported that up to $15.4 \%$ of patients admit suffering from fright during surgery with topical anesthesia. ${ }^{16}$ In the present study, both groups of patients reported similar levels of anxiety, and most of them were having only mild anxiety throughout the procedure. Anxiety during surgery may arise from pain experienced during the procedure, but the surrounding environment and the visual perception during surgery may play an important role as well. Reaffirmation from the surgeon, friendly personnel, as well as relaxing environment may help to put patient at ease and keep them calm throu ghout the surgery. The possible role of environmental factors was not examined in this study; similar ambient was created for both groups of patients by using the same medical staff for all cases.

Subjective visual sensations during cataract surgery were previously described as seeing light, various colours, movements or even instruments. ${ }^{16}$ Previous studies reported that almost $80-87 \%$ of patients with sub-tenon block perceived light during surgery, compared to $94 \%-100 \%$ of patients with topical anesthesia. ${ }^{16-19}$ In this study, patients reported similar visual sensations, which were described as seeing different colours, movements and even instruments during the procedure. Interestingly, the visual experience was not significantly reduced by the use of sub-tenon anaesthesia. Theoretically, intracameral lidocaine injection has no effect on the optic nerve, and it is thus expected that more patients in the intracameral group would experience more visual sensations compared to the regional block group. ${ }^{19}$ Chung CF et al., suggested that bright light from the microscope might induce bleaching of photoreceptors, which could, in turn, affect the vision even under intracameral anaesthesia and hence reduce the visual sensation during surgery. ${ }^{20}$

Limitations of this study include the lack of a control group, non-random samplig desing, and the use of self reporting and subjective description by the patients. Although the bias of variability had been partially eliminated by the use of standard questionnaires, pain and anxiety levels were still subjected to patient's perception, which may vary individually.

Choice of anaesthesia should always be made based on several considerations. Status of the patient and the surgeon's experience must be considered to ensure that the best choices are made. Surgeon's experience is an especially important consideration in choosing the best anaesthesia for patients undergoing complicated cataract surgery.

In conclusion, this study further supports the effectiveness of intracameral anaesthesia, as compared to sub-tenon regional anaesthesia, in maintaining patient's comfort and alleviating anxiety and pain during routine cataract surgery. Apart from akinesia, sub-tenon administration does not provide additional benefits for routine cataract surgery. Therefore, we believe that surgeons can safely select intracameral anaesthesia for patients undergoing uncomplicated cataract surgery. 


\section{References}

1. Eichel R, Goldberg I. Anaesthesia techniques for cataract surgery: A survey of delegates to the congress of the International Council of Ophthalmology, 2002. Clin Experiment Ophthalmol. 2005 0ct;33(5):469-72.

2. Zhao LQ, Zhu $H$, Zhao $P Q$, Wu QR, Hu QY. Topical anesthesia versus regional anesthesia for cataract surgery: A meta-analysis of randomized controlled trials. Ophthalmology. 2012 Apr;119(4):659-67.

3. Ezra DG, Nambiar A, Allan BD. Supplementary intracameral lidocaine for phacoemulsification under topical anaesthesia: A meta-analysis of randomized controlled trials. Ophthalmology. 2008 Mar;115(3):455-87.

4. Sreenivasa S, Zaffer R, Rodriguez A, Ksiazek S, Yee RD. Apnea and seizures following retrobulbar local anaesthetic injection. J Clin Anesth. 2003 Jun; 15(4):267-70.

5. Frieman BJ, Friedberg MA. Globe perforation associated with subtenon anaesthesia. Am J 0phthalmol. 2001 Apr;131(4):520-1.

6. Sauder $\mathrm{G}$, Jonas JB. Topical versus peribulbar anaesthesia for cataract surgery. Acta Ophthalmol Scand. 2003 Dec;81(6):596-9.

7. Combos K, Jakubovits E, Kolos A, Salacz G, Nemeth J. Cataract surgery anaesthesia really better than retrobulbar?. Acta Ophthalmol Scand. 2007 May;85(3):309-16.

8. Patel BC, Burns TA, Crandall A, Shomaker ST, Pace NL, Van EA, et al. A comparison of topical and retrobulbar anaesthesia for cataract surgery. Ophthalmology. 1996 Aug;103(8):1196-203.

9. Boezart A, Berry R, Nell M. Topical anaesthesia versus retrobulbar block for cataract surgery: The patients' perspective. J Clin Anesth. 2000 Feb;12(1):5860.

10. Rocha G, Turner C. Safety of cataract surgery under topical anaesthesia with ora sedation without anaesthetic monitoring. Can J Ophthalmol. 2007 Apr;42(2):288-94.

11. Lofoco G, Ciucci F, Bardocci A, Quercioli P, De Gaetano C, Ghirelli G, et al. Efficacy of topical plus intracameral anesthesia for cataract surgery in high myopia: Randomized controlled trial. J Cataract Refract Surg. 2008 0ct;34(10):1664-8.

12. Bijur E, Silver W, Gallagher EJ. Reliability of the visual analog scale for measurement of acute pain. Acad Emerg Med. 2001 Dec;8(12):1153-7.

13. Tseng SH, Chen FK. A randomized clinical trial of combined topical intracameral anesthesia in cataract surgery. Ophthalmology. 1998 Nov;105(11):200711.

14. Abdel-Khalek A. The development and validation of Arabic form of the STAl: Egyptian results. Pers Individ Dif. 1989;10(3):277-85.

15. Schäffer J, Mehrmann M, Heymann-Schramm S, Werry H, Piepenbrock S, et al. [Perioperative anxiety and postoperative pain suppression in intraocular operations using general anaesthesia and local anaesthesia]. Anaesthesist. 1988 Jan;37(1):19-23. German

16. Au Eong KG, Low CH, Heng WJ, Aung T, Lim TH, Ho SH, et al. Subjective visual experience during phacoemulsification and intraocular lens implantation under topical anesthesia. 0phthalmology. $2000 \mathrm{Feb} ; 107(2): 248-50$.

17. Prasad N, Kumar CM, Patil BB, Dowd TC. Subjective visual experience during phacoemulsification cataract surgery under subtenon anaesthesia. Eye (Lond). 2003 Apr;17(3):407-9.

18. Wickremasinghe SS, Tranos PG, Sinclair N, Andreou PS, Harris ML, Little BC. Visual perception during phacoemulsification cataract surgery under subtenon anaesthesia. Eye (Lond). 2003 May;17(4):501-5.

19. Ang CL, Au Eong KG, Lee SS, Chan SP, Tan CS. Patients' expectation and experience of visual sensations during phacoemulsification under topical anaesthesia. Eye (Lond). 2007 Sep;21(9):1162-7.

20. Chung CF, Lai J, Lam D. Visual sensation during phacoemulsification and intraocular lens implantation using topical and regional anesthesia. J Cataract Refract Surg. 2004 Feb;30(2):444-8.

\section{Acknowledgments}

The authors would like to acknowledge lecturers from the Department of Ophthalmology, Universiti Kebangsaan Malaysia Medical Centre for their support during the course of the study.

\section{Conflict of Interest Statement a Funding}

The Authors have no conflicts of interest to disclose. Financial support was received from the Fundamental Grant of Universiti Kebangsaan Malaysia Medical Center.

\section{Author Contributions}

Conception and design the work/idea: MLCB. Collect data/obtaining results: NSZ MHH ZAL DMD SKC SGC. Analysis and interpretation of data: MM NSZ MHH ZAL DMD SKC SGC. Write the manuscript: MM. Critical revision of the manuscript: MM MLCB. Contribution of patients or study material: MM MLCB. Obtaining financing: MM. Administrative or technical advice: MM MLCB.

Cite as:

Mustapha M, Hamzah MH, Chow SK, Zulkifli NS, Latiff ZA, Dualim DM, et al. Sub-tenon Anaesthesia versus Intracameral Anaesthesia in Patients Undergoing Cataract Extraction: A Comparative Study of the Level of Pain, Visual Perception and Anxiety. Int J Med Students. 2014 Mar-Jun;2(2):49-52. 